\title{
Uso de drogas ansiolíticas e estado nutricional de universitários
}

\author{
Drug use of anti-anxiety and nutritional status college students \\ Uso de ansiolíticos y estado nutricional de estudiantes universitarios
}

\author{
Maria da Cruz Moura e Silva \\ ORCID: https://orcid.org/0000-0002-9181-7838 \\ Universidade Federal do Piauí, Brasil \\ E-mail: mariamoura.nut@gmail.com \\ Ágatha Crystian Silva de Carvalho \\ ORCID: https://orcid.org/0000-0002-8745-6427 \\ Universidade Federal do Piaú, Brasil \\ E-mail: agathacscarvalho@hotmail.com \\ Aretha Matos de Araújo \\ ORCID: https://orcid.org/0000-0003-2305-3554 \\ Universidade Federal do Piauí, Brasil \\ E-mail: arethamatos@hotmail.com \\ Vivianne Ramos da Cunha Muniz \\ ORCID: https://orcid.org/0000-0001-8081-671X \\ Universidade Federal do Piauí, Brasil \\ E-mail: vrcmuniz@gmail.com \\ Ayla Patrícia Soares do Nascimento \\ ORCID: https://orcid.org/0000-0003-4212-0249 \\ Universidade Federal do Piauí, Brasil \\ E-mail: aylasoares10@ hotmail.com \\ Maria do Carmo Carvalho e Martins \\ ORCID: https://orcid.org/0000-0002-9107-2485 \\ Universidade Federal do Piauí, Brasil \\ E-mail: carminhacmartins@yahoo.com.br
}

\begin{abstract}
Resumo
Medicamentos ansiolíticos são drogas que agem no sistema nervoso central produzindo alterações de comportamento, humor e cognição. A prevalência do uso dessas drogas entre indivíduos jovens tem aumentado cada vez mais e isso se reflete no meio universitário, onde o uso dessas substâncias é bem difundido. O presente estudo objetivou identificar a prevalência do uso de drogas ansiolíticas entre estudantes universitários da cidade Teresina-PI e sua relação com o estado nutricional. Trata-se de estudo transversal com 665 universitários, que envolveu o preenchimento de um questionário para identificação do uso de ansiolíticos e aferição das medidas antropométricas de peso e estatura para classificação do estado nutricional por meio do índice de massa corporal. As associações entre as variáveis foram feitas aplicando-se o teste do qui-quadrado $(\mathrm{p}<0,05)$. O uso de ansiolíticos foi referido por $8,4 \%$ dos estudantes, dos quais, a maioria era mulheres, sendo que $76,7 \%$ o faziam com indicação médica. As drogas mais utilizadas foram diazepam $(51,8 \%)$ e clonazepam $(12,5 \%)$. Com relação ao estado nutricional, $81,8 \%$ se encontravam eutróficos. A prevalência do uso de drogas ansiolíticas entre os universitários foi elevada. Contudo, não houve associação entre seu uso e o estado nutricional, sugerindo que essas drogas parecem não interferir no estado nutricional.
\end{abstract}

Palavras-chave: Estudantes; Ansiolíticos; Estado nutricional; Ansiedade.

\begin{abstract}
Anxiolytic drugs are drugs that act on the central nervous system producing changes in behavior, mood and cognition. The prevalence of the use of these drugs among young individuals has increased more and more and this is reflected in the university environment, where the use of these substances is widespread. The present study aimed to identify the prevalence of the use of anti-anxiety agents among college students in the city of Teresina-PI and its relation with nutritional status. This is across-sectional study with 665 college students, who filled out a questionnaire to identify the us e of anti-anxiety agents and underwent anthropometric measure men to height and weight to determine nutritional status. The associations among the different variables were made through the use of the chi-square test (p $<0,05)$. The use of anti-anxiety agents was referred to by $8,4 \%$ of the students, from which most where women, while $76,7 \%$ of these cases corresponded to medical prescription. The most common drugs were diazepam $(51,8 \%)$ and clonazepam (12,5\%). Regarding the nutritional status, $81,8 \%$ were eutrophic. The prevalence of use of anti-anxiety agents was high among college students. However, there was no association between their use and the nutritional status, suggesting that these drugs seem not to interfere in the nutritional status.
\end{abstract}

Keywords: Students; Anti-anxiety agents; Nutritional status; Anxiety. 


\begin{abstract}
Resumen
Los ansiolíticos son fármacos que actúan sobre el sistema nervioso central produciendo cambios en el comportamiento, el estado de ánimo y la cognición. La prevalencia del uso de estas drogas entre los jóvenes ha aumentado cada vez más y esto se refleja en el ámbito universitario, donde el uso de estas sustancias está muy extendido. El presente estudio tuvo como objetivo identificar la prevalencia del consumo de ansiolíticos en estudiantes universitarios de la ciudad de Teresina-PI y su relación con el estado nutricional. Se trata de estudio transversal con 665 estudiantes universitarios, que implicó completar un cuestionario para identificar el uso de ansiolíticos y hacer medidas antropométricas de peso y talla para clasificar el estado nutricional mediante el índice de masa corporal. Las asociaciones entre variables se realizaron mediante la prueba de chi-cuadrado (p <0,05). El uso de ansiolíticos fue reportado por el 8,4\% de los estudiantes, la mayoría mujeres, y el 76,7\% lo hizo con recomendación médico. Los fármacos más utilizados fueron diazepam $(51,8 \%)$ y clonazepam $(12,5 \%)$. En relación al estado nutricional, $81,8 \%$ estaban eutróficos. La prevalencia del consumo de ansiolíticos entre los estudiantes universitarios fue alta. Sin embargo, no hubo asociación entre su uso y el estado nutricional, lo que sugiere que estos medicamentos no parecen interferir con el estado nutricional.
\end{abstract}

Palabras clave: Estudiantes; Ansiolíticos; Estado nutricional; Ansiedad.

\title{
1. Introdução
}

Os medicamentos ansiolíticos são drogas psicoativas e psicotrópicas. Droga psicoativa é aquela que pode alterar o comportamento, o humor e a cognição, e psicotrópica é aquela que age no sistema nervoso central produzindo alterações de comportamento, humor e cognição, com grande propriedade reforçadora (Carlini et al., 2001). Vários fatores estão envolvidos no uso de medicamentos psicotrópicos, incluindo estresse, depressão, ansiedade, insônia e problemas sociais, dentre outros. Sua utilização prolongada pode favorecer a tolerância e a dependência, sendo essa última decorrente da interação entre dose, duração do tratamento, regularidade do uso, droga utilizada e perfil psicológico do paciente (Carlini et al., 2001; Margarido, 2012; Telles Filho et al., 2011; Nascimento \& Sakata, 2011).

Os medicamentos psicotrópicos, especialmente os da classe dos ansiolíticos, estão entre os mais consumidos pela população adulta. A prevalência do uso dessas drogas entre indivíduos jovens tem aumentado cada vez mais e isso se reflete no meio universitário, onde o uso dessas substâncias é bem difundido (Wanscher et al, 2014).

A graduação universitária é um período que conta com diversos fatores ansiogênicos. A apreensão acerca do futuro é um componente contínuo que pode contribuir significantemente para o desenvolvimento da ansiedade nesse grupo. Esse período, ainda que traga sentimentos positivos e de alcance de uma meta programada, por vezes, pode se tornar crítico e de maior vulnerabilidade para o início e a manutenção do uso de psicotrópicos (Marchi et al, 2013).

O consumo abusivo de drogas causa danos físicos, psíquicos e emocionais em todas as faixas etárias. Contudo, é entre crianças e adolescentes que seus efeitos são mais deletérios para o desenvolvimento biopsicossocial. Os adolescentes constituem-se no grupo populacional mais vulnerável ao uso e abuso de drogas, e as consequências negativas geradas no desenvolvimento biopsicossocial são objeto de preocupações entre diferentes profissionais, sobretudo os da área da saúde. Uma das problemáticas vinculadas ao uso de drogas entre adolescentes está relacionada à questão nutricional, que tem relevância no desenvolvimento destes indivíduos, uma vez que seu organismo encontra-se em pleno processo de crescimento e amadurecimento orgânico (Cozer \& Gouvêa, 2010).

Estudo realizado com jovens universitários em Teresina-PI mostrou que o consumo de substâncias psicotrópicas nesse grupo é uma realidade preocupante, visto que boa parte já consumiu alguma substância ilícita, principalmente indivíduos do sexo masculino (Martins et al., 2012). Ademais, o uso frequente dessas substâncias em adolescentes e adultos jovens tem sido relacionado a um conjunto de consequências deletérias, tais como acidentes automobilísticos, violência, comportamento sexual de risco, prejuízos acadêmicos, diminuição da percepção e estresse (Teixeira et al., 2009).

No Brasil, os estudos que avaliam o uso de drogas psicotrópicas pelos jovens e sua relação com o estado nutricional ainda são incipientes. Nesse contexto, é interessante esclarecer possíveis interferências do consumo dessas drogas sobre a situação nutricional desses indivíduos, visto que diversos malefícios por sua utilização têm sido referidos. Assim, o presente 
estudo teve como objetivo identificar a prevalência de uso de drogas ansiolíticas entre estudantes universitários da cidade Teresina-PI e sua relação com o estado nutricional.

\section{Metodologia}

Este trabalho fez parte do projeto de pesquisa intitulado "Perfil socioeconômico, nutricional e de saúde de estudantes da Universidade Federal do Piauí (UFPI) - Brasil" e consistiu em um estudo quantitativo e descritivo, com delineamento transversal, realizado com estudantes de todos os cursos regulares de graduação da UFPI. O método quantitativo trabalha com a representatividade numérica da população e quantificação dos resultados (Zanella, 2013).

A amostra foi calculada considerando nível de confiança de $95 \%$ e população finita de 11.152 estudantes, segundo a fórmula utilizada por Martins et al., (2002) para estimativas da proporção para grandes amostras e margem de erro de 3\% para mais ou para menos. A amostra foi estratificada por meio de uma partilha proporcional que considerou centro de ensino, curso, período cursado e turno (noturno/diurno). Participaram do estudo 665 estudantes (310 homens e 355 mulheres) com média de idade de $21,85 \pm 4,2$ anos.

A coleta de dados foi feita utilizando-se questionário auto preenchível e não identificado, baseado no estudo realizado pelo Centro Brasileiro de Informações sobre Drogas Psicotrópicas (CEBRID), e incluiu questões sobre uso de ansiolíticos e também para obtenção de dados socioeconômicos (idade, sexo, estado civil, renda familiar e grau de escolaridade da mãe). Além disso, também foram obtidas medidas antropométricas de peso corporal (aferido em balança digital com capacidade de a $150 \mathrm{~kg}$ e variação de $0,1 \mathrm{~kg}$ ) e estatura (medida com fita antropométrica, com precisão de $1 \mathrm{~mm}$ ) realizadas com os estudantes descalços, usando roupas leves e sem acessórios que pudessem interferir nas medidas, seguindo as recomendações do Manual de Técnicas e Procedimentos do Ministério da Saúde (Brasil, 2003).

O estado nutricional global foi classificado a partir do índice de massa corporal (IMC) com base nos pontos de corte propostos pela World Health Organization (WHO, 1998), sendo eutrofia definida como IMC $>18,5 \mathrm{e}<25 \mathrm{~kg} / \mathrm{m}^{2}$, sobrepeso como IMC $\geq 25 \mathrm{e}<30 \mathrm{~kg} / \mathrm{m}^{2}$ e obesidade como IMC $\geq 30 \mathrm{~kg} / \mathrm{m}^{2}$.

O projeto foi aprovado pelo Comitê de Ética em Pesquisa da UFPI (Parecer $n^{\circ}$ 95/2006). Os participantes foram previamente informados a respeito da pesquisa e assinaram um termo de consentimento livre e esclarecido, de acordo com as diretrizes da resolução ou 196/96do Conselho Nacional de Saúde.

Os dados foram processados nos programas Excel for Windows e SPSS (Statiscal Package for the Sciences) versão 20.0. A análise estatística para associações entre as variáveis estudadas foi realizada por meio da aplicação do teste de associação do qui-quadrado. O nível de significância foi estabelecido em $\mathrm{p}<0,05$.

\section{Resultados}

Na Tabela 1 é apresentada a distribuição dos participantes no estudo segundo as características sociodemográficas e a classificação do estado nutricional. Pouco mais de metade dos estudantes, $(53,4 \%)$ era do sexo feminino, sendo a maioria solteira (94,9\%), e na faixa etária de 16 a 25 anos (86,5\%). Verificou-se também que 55,5\% dos estudantes exerciam alguma atividade de trabalho remunerado, e que apenas 19,2\% eram filhos de mulheres com curso superior completo. Metade dos estudantes pertencia a famílias com renda mensal igual ou superior a seis salários mínimos vigentes na época, e, apenas $0,5 \%$ dos pesquisados, pertenciam a famílias com renda mensal menor que um salário mínimo vigente na época. No tocante ao estado nutricional, a maioria $(81,8 \%)$ encontrava-se eutrófica. 
Tabela 1 - Distribuição da população estudada segundo uso de drogas ansiolíticas. Teresina-PI, 2014.

\begin{tabular}{|c|c|c|c|c|c|c|c|c|}
\hline \multirow[t]{3}{*}{ Variáveis } & & & & \multicolumn{4}{|c|}{ Uso de Ansiolíticos } & \multirow[b]{3}{*}{$\mathbf{p}^{*}$} \\
\hline & & \multicolumn{2}{|c|}{ Prevalência } & \multicolumn{2}{|c|}{ Sim } & \multicolumn{2}{|c|}{ Não } & \\
\hline & & $\mathbf{n}$ & $\%$ & $\mathbf{n}$ & $\%$ & $\mathbf{n}$ & $\%$ & \\
\hline \multirow[t]{2}{*}{ Sexo } & Masculino & 310 & 46,6 & 17 & 5,5 & 293 & 94,5 & 0,011 \\
\hline & Feminino & 355 & 53,4 & 39 & 11 & 316 & 89,9 & \\
\hline \multirow{4}{*}{$\begin{array}{l}\text { Faixa } \\
\text { (anos) }\end{array}$} & $16-25$ & 575 & 86,5 & 46 & 8 & 529 & 92 & 0,448 \\
\hline & $26-30$ & 69 & 10,4 & 9 & 13 & 60 & 87 & \\
\hline & $31-40$ & 15 & 2,3 & 1 & 6,7 & 14 & 93,3 & \\
\hline & $>40$ & 6 & 0,9 & 0 & 0 & 6 & 100 & \\
\hline \multirow{2}{*}{$\begin{array}{l}\text { Trab.remunerad } \\
\text { o }\end{array}$} & Sim & 369 & 55,5 & 30 & 8,1 & 339 & 91,9 & 0,763 \\
\hline & Não & 296 & 44,5 & 26 & 8,8 & 270 & 91,2 & \\
\hline \multirow{5}{*}{$\begin{array}{l}\text { Renda familiar } \\
\text { mensal } \\
\left(\mathrm{SM}^{* *}\right)\end{array}$} & $\leq 1$ & 3 & 0,5 & 0 & 0 & 3 & 100 & 0,764 \\
\hline & $>1 \mathrm{e} \leq 2,5$ & 168 & 25,3 & 13 & 7,7 & 155 & 92,3 & \\
\hline & $>2,5 \mathrm{e} \leq 6,5$ & 162 & 24,4 & 12 & 7,4 & 150 & 92,6 & \\
\hline & $>6,5 \mathrm{e} \leq 8$ & 178 & 26,8 & 19 & 10,7 & 159 & 89,3 & \\
\hline & $>8$ & 154 & 23,2 & 12 & 7,8 & 142 & 92,2 & \\
\hline \multirow[t]{4}{*}{ Estado civil } & Solteiro & 631 & 94,9 & 51 & 8,1 & 580 & 91,9 & 0,540 \\
\hline & Casado & 9 & 1,3 & 1 & 11,1 & 8 & 88,9 & \\
\hline & Divorciado & 14 & 2,1 & 2 & 14,3 & 12 & 85,7 & \\
\hline & Outros & 11 & 1,7 & 2 & 18,2 & 9 & 81,8 & \\
\hline \multirow{3}{*}{$\begin{array}{l}\text { Estado } \\
\text { nutricional }\end{array}$} & Eutrofia & 544 & 81,8 & 47 & 8,6 & 497 & 91,3 & 0,758 \\
\hline & Sobrepeso & 103 & 15,5 & 7 & 6,8 & 96 & 93,2 & \\
\hline & Obesidade & 18 & 2,7 & 2 & 11,1 & 16 & 88,9 & \\
\hline
\end{tabular}

Não houve associação estatisticamente significativa entre o uso de ansiolíticos e o estado nutricional, estado civil, renda familiar, idade, grau de escolaridade da mãe e possuir trabalho remunerado (Tabela 1). Porém, foi observada associação entre sexo feminino e o uso de drogas ansiolíticas $(\mathrm{p}=0,011)$.

A Tabela 2 mostra o percentual de uso de ansiolíticos, bem como os mais utilizados entre os universitários.

Tabela 2 - Uso de ansiolíticos pelos estudantes universitários. Teresina-PI, 2014.

\begin{tabular}{lcc}
\hline \multicolumn{1}{c}{ Variável } & $\mathrm{n}$ & $\%$ \\
\hline Usa ou já usou ansiolítico?* & & \\
Sim & 56 & 8,4 \\
Não & 609 & 91,6 \\
Quem indicou o medicamento?** & & 76,7 \\
Médico & 43 & 5,3 \\
Outro profissional de saúde & 3 & 3,6 \\
Balconista de farmácia & 2 & 25,0 \\
Parente ou amigo & 14 & 12,5 \\
Iniciativa própria & 7 & 51,8 \\
Qual droga ansiolítica utilizou ou utiliza?** & & 12,5 \\
Diazepam & 29 & 1,8 \\
Clonazepam & 7 & 1,8 \\
Lorazepam & 1 & 8,9 \\
Bromazepam & 1 & 46,4 \\
Mais de um tipo & 5 & 26 \\
Outros & 26 \\
\hline
\end{tabular}

*Pergunta direcionada a todos os participantes da pesquisa,** pergunta direcionada aos indivíduos que usavam ou usaram drogas ansiolíticas.

O uso de ansiolíticos foi referido por 8,4\% dos estudantes e destes,76,7\% o faziam com indicação médica. Os demais referiram uso por iniciativa própria, indicação de parente ou amigo, balconista de farmácia ou outro profissional de saúde. Os 
ansiolíticos mais utilizados entre os estudantes foram o diazepam $(51,8 \%)$ e o clonazepam (12,5\%). Além destes, outros tipos também eram utilizados $(46,4 \%)$, ressaltando-se que alguns dos estudantes utilizavam mais de um tipo de ansiolítico.

\section{Discussão}

Com relação às características sociodemográficas, observou-se que a maioria dos universitários avaliados exercia algum tipo de atividade remunerada, fatores, que por sua vez, podem ter contribuído para o acesso dos estudantes às drogas ansiolíticas. Além disso, verificou-se maior prevalência de uso dessas drogas entre os universitários com idades de 16 a 25 anos, perfil também encontrado em estudo realizado com acadêmicos do curso de odontologia da Universidade Federal do Espírito Santo (Teixeira et al., 2010), e também entre universitários de El Salvador (Ortega-Pérez et al., 2011), assim como entre universitários baianos (Souza et al., 2021).

Destaca-se ainda a associação entre uso de ansiolíticos e sexo feminino. Dados semelhantes também foram obtidos no estudo de Botti et al. (2010), realizado com estudantes do curso noturno de enfermagem da Pontifícia Universidade Católica de Minas Gerais, no qual houve prevalência de 19,94\% de uso entre as universitárias; enquanto para os estudantes do sexo masculino foi de 11,90\%. Por outro lado, no estudo de Teixeira et al., (2009), realizado com estudantes do curso de odontologia da Universidade Federal do Espírito Santo, essa diferença não foi observada, mas houve prevalência semelhante à obtida no presente estudo. Em contrapartida, a pesquisa realizada por Martins et al. (2012), demonstrou que a maioria dos consumidores de drogas psicotrópicas, entre universitários, era do sexo masculino, com 26,2\% de prevalência.

Entre as razões que poderiam explicar o fato de jovens, independentemente do sexo, usarem drogas ilícitas estão: prazer, pressão social, curiosidade, hábito e estresse (Romero et al., 2009). No caso de estudantes universitários, essa realidade não é diferente, sendo que o estresse e a ansiedade figuram entre os motivos para uso de substâncias psicoativas por esse público (Ortega-Pérez et al., 2011).

A prevalência do uso de ansiolíticos foi de $8,4 \%$ (Tabela 2). Resultados semelhantes foram encontrados por Lucas $e t$ al. (2006) e Canuto et al. (2006), em que as prevalências foram de 9,2\% e 7,7\%, respectivamente. Por outro lado, Botti et al. (2010), constataram que entre as drogas ilícitas de uso mais frequente entre acadêmicos de enfermagem estavam os ansiolíticos, com 19,08\%. E Marchi et al. (2013) também pesquisando estudantes de enfermagem verificaram que 16\% utilizavam esses medicamentos. Tal diferença pode ter ocorrido pelo fato de o presente estudo ter incluído todos os universitários, não levando em conta o curso que faziam, enquanto os estudos citados analisaram apenas graduandos da área da saúde, que podem ter facilidade de acesso a tais medicamentos, além de conhecimento acerca de seus efeitos.

Entre os fatores associados ao uso de ansiolíticos nesse grupo populacional pode-se citar a gestão de um grande número de informações, problemas relacionados ao ambiente educacional, ansiedade relacionada ao ingresso no mercado de trabalho, além de fatores pessoais como problemas familiares, conflitos envolvendo relacionamentos afetivos e vulnerabilidades psicológicas (Dehghan-nayeri \& Adib-Hajbaghery, 2011; Galindo et al. 2009).

Entre os $8,4 \%$ de universitários que utilizavam as drogas ansiolíticas, $76,7 \%$ deles afirmaram fazê-la sob prescrição médica, resultado similar ao obtido no estudo de Souza et al. (2021). Já 23,3\% deles que relataram fazer auto-medicação, sendo este um fato interessante a ser observado, já que é de conhecimento público que esses medicamentos somente podem ser vendidos sob prescrição médica. Ressalta-se, ainda, que essas drogas apresentam uma série de efeitos colaterais ao organismo humano, quando utilizadas indiscriminadamente, além de causarem dependência química e dificuldade na interrupção do tratamento.

A maior parte dos estudantes que referiu uso de ansiolíticos, afirmou consumir mais de um tipo desses medicamentos, sendo os mais apontados o diazepam e o clonazepam. Resultados semelhantes foram encontrados em acadêmicos de enfermagem da Universidade Católica de Minas Gerais (Botti \& Simões, 2010) e no estudo de Bauchrowitz et al. (2019). É 
importante ressaltar que os ansiolíticos possuem efeitos adversos que podem ser potencializados pelo uso concomitante de outros psicotrópicos, bem como maior chance de causar forte dependência (Firmino et al., 2011), situação frequente entre os estudantes usuários no presente estudo.

Não foi encontrada associação entre o consumo de drogas e o estado nutricional, e a maioria dos estudantes $(81,8 \%)$ apresentou-se eutrófica. Tal fato sugere que, apesar de serem inúmeros os prejuízos causados pelo uso de ansiolíticos no grupo estudado, eles parecem não afetar o estado nutricional, porém, outros fatores devem ser investigados, como a prática de atividade física regular e o tipo de alimentação feita pelos universitários. Acredita-se que boa parte desses indivíduos, devido à rotina exaustiva de estudos e por terem aulas em tempo integral, realize com regularidade suas refeições em restaurantes universitários, conseguindo ter uma alimentação balanceada. Essa situação, aliada à prática de atividade física regular poderia proporcionar uma vida mais saudável, evitando alterações do estado nutricional.

Uma limitação encontrada no presente estudo refere-se ao fato de o mesmo não ter avaliado os fatores que poderiam ser associados à prevalência do uso de drogas ansiolíticas e sua relação com o estado nutricional, bem como por ter avaliado os universitários sem considerar o curso de graduação que faziam, já que é apontado pela literatura, que existem diferenças possivelmente relacionadas a isso. Dessa maneira, mais estudos devem ser desenvolvidos com esta população, a fim de investigar mais profundamente os motivos relacionados ao uso de ansiolíticos, bem como sanar as limitações citadas.

\section{Conclusão}

Considera-se elevada a prevalência do uso de ansiolíticos na população estudada, o que representa um risco à saúde, devido à propensão deste grupo à utilização contínua de tais medicamentos, que, em longo prazo, podem gerar dependência química. Assim, evidencia-se a necessidade de ações de educação em saúde, voltadas para esse público, com o objetivo de esclarecer sobre os riscos do uso indiscriminado dessas drogas.

Nesse estudo, não foi verificada associação entre o uso de ansiolíticos e o estado nutricional, mostrando que essas drogas parecem não interferir nessa variável no grupo estudado. Sugere-se a realização de mais estudos que analisem outros fatores que também possam influenciar essa relação, como a prática regular de atividade física, os hábitos alimentares e o tempo de uso do ansiolítico.

\section{Referências}

Botti, N. C. L., Lima, A. F. D., \& Simões, W. M. B. (2010). Uso de substâncias psicoativas entre acadêmicos de enfermagem da universidade católica de Minas Gerais. Revista Electrónica em Salud Mental, Alcohol y Drogas, 6(1): 1-13.

Brasil. Ministério da Saúde. (2003). Universidade Federal de Goiás. Centro Colaborador em Alimentação e Nutrição da Região Centro-Oeste. Antropometria. Manual de técnicas e procedimentos. Vigilância Nutricional. (2a ed.).

Bauchrowitz, C., Paz. L. E. C., Müller, E. V., Possagno, G. C. H., \& Minozzo, B. R. (2019). Prevalência de uso de psicofármacos por acadêmicos: efeitos do processo de graduação. Braz. J. of Develop.5(11): 24915-24933.

Canuto, M. H. A., Ferreira, R. A., \& Guimarães, E. M. B. (2006). Uso e abuso de drogas ilícitas por jovens do $1^{\text {o }}$ ano da Universidade Federal de Goiás. Revista Paulista de Pediatria, 24(2): 135-42.

Carlini, E. A., Nappo, S. A., Galduróz, J. C. F., \& Noto, A. R. (2001). Drogas psicotrópicas: O que são e como agem. Revista IMESC; 1(3):9-35.

Cozer, M. \& Gouvêa, L. A. V. N. (2010). Avaliação do estado nutricional e hábito alimentar de adolescentes frequentadores do CAPS AD de um município do oeste do Paraná. Rev Tempus Actas Saúde Colet, 4(1):145-54.

Dehghan-nayeri, N., \& Adib-Hajbaghery, M. (2011). Effects of progressive relaxation on anxiety and quality of life in female students: A non-randomized controlled trial. Alternat Complement Ther,19:194-200.

Firmino, K. F., Abreu, M. H. N. G., Perini, E., \& Magalhães, S. M. S. (2011). Fatores associados ao uso de benzodiazepínicos no serviço municipal de saúde da cidade de Coronel Fabriciano, Minas Gerais, Brasil. Cad. Saúde Pública, 27(6) 6:1223-1232.

Galindo, S. B., Moreno, I. M., \& Muñoz, J. G. (2009). Prevalencia de andiedad y Depressión em uma población de Estudiantes universitários: Factores acadêmicos y sociofamiliaresasociados. Clínica y Salud, 20(2):177-187. 
Research, Society and Development, v. 10, n. 16, e141101623254, 2021

(CC BY 4.0) | ISSN 2525-3409 | DOI: http://dx.doi.org/10.33448/rsd-v10i16.23254

Lucas, A. C. S., Parente, R. C. P., Picanço. N. S., Conceição, D. A., Costa, K. R. C., Magalhães, R. S., \& Siqueira, J. C. (2006). Uso de psicotrópicos entre universitários da área da saúde da Universidade Federal do Amazonas, Brasil. Cad. Saúde Pública, 22(3):663-671.

Marchi, K. C., Bárbaro, A. M., Miasso, A. I., \& Tirapelli, C. R. (2013). Ansiedade e consumo de ansiolíticos entre estudantes de enfermagem de uma universidade pública. Rev. Eletr. Enf., 5(3):731-9.

Margarido, F. B. (2012). A banalização do uso de ansiolíticos e antidepressivos. Rev. Psicolog, 15(22):131-146.

Martins, G. A. Estatística geral e aplicada. (2002) (2a ed.), Atlas.

Martins, M. C. C., Filho, M. D. S., Santos, T. L., Sousa, L. G., Carvalho, I. L. N. F., Silva, R. O et al. (2012). Uso de drogas psicotrópicas ilícitas entre os estudantes de uma universidade pública.Brasília Med, 49(3):150-157.

Nascimento, D. C. H., \& Sakata, R. K. (2011). Dependência de opioide em pacientes com dor crônica. Rev Dor;12(2):160-5.

Ortega-Pérez, C. A., Costa-Júnior, M. L., \& Vasters, G. P. (2011). Perfil epidemiológico de la drogadicción em estudiantes universitários. Rev. Latino-Am. Enfermagem, 19:665-72.

Romero, M. I., Jaime, Santander, J. Hitschfeld, M. J., Labbé, M., \& Zamora, V. (2009). Consumo de sustancias ilícitas y psicotrópicos entre los Estudiantes de medicina de laPontificiaUniversidad Católica de Chile. RevMéd Chile, 137: 459-465.

Souza, M. S. P., Almeida, R. L. M. L., Amorim, A. T., \& Santos, T. A. (2021). Uso de antidepressivos e ansiolíticos entre estudantes do curso de farmácia em uma instituição privada e uma pública do interior da Bahia. Research, Society and Development,10(8):e29610817177

Teixeira, A. F., Aliane, P. P., Ribeiro, L. C., \& Ronzani, T. M. (2009). Uso de substâncias psicoativas entre estudantes de Goianá, MG. Est Psicol, 14(1): 5157.

Teixeira, R. F., Souza, R. S., Buaiz, V., \& Siqueira, M. M. (2010). Uso de substâncias psicoativas entre estudantes de odontologia da Universidade Federal do Espírito Santo. Cien\& Saúde Colet, 15(3):655-662.

Telles Filho, P. C. P., Lima, A. M. J.,Chagas, A. R., Durão, A. M. S., \& Pinheiro, M. L. P. (2011). Utilização de benzodiazepínicos por idosos de uma estratégia de saúde da família: implicações para enfermagem. Escola Anna Nery,15(3): 581-586.

Wansche, D., Prado, G. P., \& Frigo, J. (2014). Uso de psicotrópicos por alunos do ensino superior. Rev. UNINGÁ Review,18(2):05-09.

World Health Organization. (1998). Obesity: preventing and managing the global epidemic: report of a WHO consultation on obesity. Geneva.

Zanella, L. C. H. (2013). Metodologia de pesquisa. (2a ed.). Departamento de Ciências da Administração/UFSC. 